\title{
VLT-UVES spectroscopy of a bulge giant magnified through microlensing: EROS-BLG-2000-5^
}

\author{
D. Minniti ${ }^{1}$, B. Barbuy ${ }^{2}$, V. Hill ${ }^{3}$, E. Bica ${ }^{4}$, S. Ortolani ${ }^{5}$, R. M. Rich ${ }^{6}$, K. H. Cook $^{7}$, T. Vandehei ${ }^{8}$, \\ A. Renzini ${ }^{9}$, L. Pasquini ${ }^{9}$, and D. Bennett ${ }^{10}$ \\ 1 Universidad Catolica de Chile, Department of Astronomy \& Astrophysics, Casilla 306, Santiago 22, Chile \\ e-mail: dante@astro.puc.cl \\ 2 Universidade de São Paulo, Departamento de Astronomia, CP 3386, São Paulo 01060-970, Brazil \\ 3 Observatoire de Paris-Meudon, 92195 Meudon Cedex, France \\ e-mail: Vanessa.Hill@obspm.fr \\ 4 Universidade Federal do Rio Grande do Sul, Departamento de Astronomia, CP 15051, Porto Alegre 91501-970, \\ Brazil \\ e-mail: bica@if.ufrgs.br \\ 5 Università di Padova, Dipartimento di Astronomia, Vicolo dell'Osservatorio 2, 35122 Padova, Italy \\ e-mail: ortolani@pd.astro.it \\ ${ }^{6}$ UCLA, Department of Physics \& Astronomy, 8979 Math-Sciences Building, Los Angeles, CA 90095-1562, USA \\ e-mail: rmr@astro.ucla.edu \\ 7 Lawrence Livermore National Laboratory, Livermore, CA 94550, USA \\ e-mail: kcook@llnl.gov \\ 8 University of California, Department of Physics, San Diego, CA 92093, USA \\ e-mail: vandehei@astrophys.ucsd.edu \\ 9 European Southern Observatory, Karl Schwarzschild Strasse 2, 85748 Garching bei München, Germany \\ e-mail: arenzini@eso.org, lpasquin@eso.org \\ 10 University of Notre Dame, Department of Physics, Notre Dame, IN 46556, USA \\ e-mail: bennett@bustard.phys.nd.edu
}

Received 28 November 2001 / Accepted 15 January 2002

\begin{abstract}
High resolution spectra of a magnified bulge star were obtained at the $8 \mathrm{~m}$ Kueyen VLT-UT2 telescope with the UVES spectrograph. The source is a bulge giant of baseline magnitude $V_{\mathrm{EROS}}=18.4$, magnified to $V \approx 18.0$. These data allow us to derive the stellar parameters of effective temperature $T_{\text {eff }} \sim 3800 \mathrm{~K}$, gravity $\log g \sim 1.0$, reddening $E(B-V) \sim 0.7$, metallicity $[\mathrm{Fe} / \mathrm{H}] \sim-0.3$ and and $[\mathrm{O} / \mathrm{Fe}] \approx 0.0$ for EROS-BLG-2000-5. The giant appears to be located in the far side of the bulge.
\end{abstract}

Key words. Galaxy: bulge - abundances - gravitational lensing

\section{Introduction}

A major contribution of microlensing surveys is the possibility of magnifying faint stars, in particular in the Galactic bulge where, due to crowding, a number of alerts are detected. Very few spectroscopic data have taken advantage of the magnification. Published studies so far are

Send offprint requests to: B. Barbuy,

e-mail: barbuy@iagusp.usp.br

* Observations collected at the European Southern Observatory - ESO, Paranal, Chile; ESO proposal No. 65.L0340 . those by Benetti et al. (1995) who detected a G/K subgiant bulge star; Lennon et al. (1996) used the NTT and EMMI spectrograph to observe the event 96-BLG-3 revealing it to be a G0 IV-V star of metallicity $[\mathrm{M} / \mathrm{H}]=$ +0.3 to +0.6 , and Minniti et al. (1998) used the Keck telescope to observe the event 97-BLG-45, corresponding to a main sequence bulge star, and determined a lithium abundance of $N(\mathrm{Li})=2.25$.

During observations on a program at the VLT to obtain high resolution spectra of bulge field stars, with priority to microlensed objects, we concentrated efforts on the alert event EROS-BLG-2000-5. The first caustic peak 
occurred on June 14, 2000 (HJD 2451705), and the second caustic peak on July 4, 2000 (HJD 2451730). We observed this event in between peaks, on June 24-25, 2000 (HJD 2451719). Previous studies of the same event, with the aim of studying its magnitude, colour and $\mathrm{H} \alpha$ variability in timescales of hours or days, and mass measurement, were presented by Castro et al. (2001), Albrow et al. (2001) and An et al. (2001).

The star is located at J2000 $\alpha=17^{\mathrm{h}} 53^{\mathrm{m}} 11.5^{\mathrm{s}}, \delta=$ $-30^{\circ} 55^{\prime} 35^{\prime \prime}\left(l=359.1356^{\circ}, b=-2.4299^{\circ}\right)$. Finding charts and light curves are available from the EROS Alert webpage: http://www-dapnia.cea.fr/Spp/Experiences/ EROS/e-blg-2000-5/index.html.

We are dealing with a $V_{\text {EROS }}=18.4,(V-R)_{\mathrm{EROS}}=$ 1.2 star (where these magnitudes correspond to EROS measurements calibrated to Cousins colours), that is in the middle of a binary microlensing event, and was magnified by about 0.4 magnitudes during our run. A model of this event indicates that it is blended with a much redder star, and this redder star is likely to be the lens star. The blended star that is not lensed is 0.6 mag redder in a very sparse region of the Colour-Magnitude Diagram (CMD), suggesting that the lens is an M dwarf and the source is a $\mathrm{K}$ giant.

The red giant branch star NGC 6528: I-2 (cf. notation by van den Bergh \& Younger 1979) belonging to the metalrich bulge globular cluster NGC 6528 located at J2000 $\alpha=$ $18^{\mathrm{h}} 04^{\mathrm{m}} 51.5^{\mathrm{s}}, \delta=-30^{\circ} 03^{\prime} 04^{\prime \prime}\left(l=1.1459^{\circ}, b=-4.1717^{\circ}\right)$, is also presented here for comparison purposes.

In the present paper, we make an effort to determine the atmospheric parameters of the lensed star, in view of contributing to future modeling of the event.

In Sect. 2 the observations are described. In Sect. 3 the stellar parameters and oxygen abundance are deduced. Concluding remarks are given in Sect. 4.

\section{Observations}

High resolution spectra in the wavelength range $\lambda \lambda 4800$ $6800 \AA$ were obtained with the UVES spectrograph at the VLT UT2 telescope at ESO (Paranal).

Three exposures of 1.5 hours each for EROS-BLG2000-5 were obtained on 2000 June 24-25.

The spectrum of the cool giant NGC 6528: I-2 was also observed with the same instrumentation and configuration.

With the standard configuration, a resolution of $R \sim$ 45000 was achieved for a slit width of $1.0^{\prime \prime}$ for EROS-BLG2000-5 and $R \sim 55000$ for a slit of $0.8^{\prime \prime}$ for NGC 6528: I-2. The log of observations is provided in Table 1 . The $S / N$ reported in Col. 3 refers to that measured per pixel of $0.017 \AA$.

The spectra were reduced using the UVES context of the MIDAS reduction package, including bias and interorder background subtraction, flatfield correction, optimal extraction (with cosmic ray rejection) above the sky level and wavelength calibration (Balester et al. 2000).
Table 1. Log of observations carried out on 2000, June 24-25. Three exposures of 1.5 hours were obtained for EROS-BLG2000-5 and two exposures of 1.5 hours for NGC 6528: I-2. Mean seeing values over the exposures are given.

\begin{tabular}{lllll}
\hline Target & ST & Seeing $\left(^{\prime \prime}\right)$ & Airmass & $S / N$ \\
\hline NGC 6528: I-2 & $00: 04: 17$ & 1.3 & 1.9 & 50 \\
& $01: 29: 52$ & 1.2 & 1.3 & 55 \\
\hline EROS-BLG-2000-5 & $03: 01: 11$ & 1.1 & 1.1 & 20 \\
& $04: 32: 30$ & 1.0 & 1.0 & 16 \\
& $06: 10: 56$ & 1.1 & 1.1 & 16 \\
\hline
\end{tabular}

\section{Spectroscopic analysis}

Figure 1 shows the data of EROS-BLG-2000-5 (upper spectrum) and NGC 6528: I-2 (lower spectrum), heavily smoothed to show the flux distribution of the two stars. Note the striking similarity of the two spectra, and in particular, the TiO bands near $6150 \AA$ and longer wavelengths. The spectra were flux calibrated using a spectrophotometric standard taken on June 26, corrected for airmass (Geneva coefficients for La Silla), and then normalised arbitrarily to a flux of unity at $5870 \AA$ ( $V$ band) in order to compare them.

We do not see any evidence of the lensing $M$ dwarf in the spectrum, such as double lines. We do detect TiO, at the radial velocity of the $\mathrm{K}$ giant itself, which suggests that we are dealing with a late $\mathrm{K}$ or early $\mathrm{M}$ giant star.

A radial velocity of $v_{\mathrm{r}}=-191 \mathrm{~km} \mathrm{~s}^{-1}$ is obtained for EROS-BLG-2000-5. This is consistent with bulge membership, being at the high velocity tail of the bulge velocity distribution (Minniti 1996; Ibata \& Gilmore 1995). This velocity also rules out the possibility of this star belonging to the Sgr dwarf galaxy (Ibata et al. 1995).

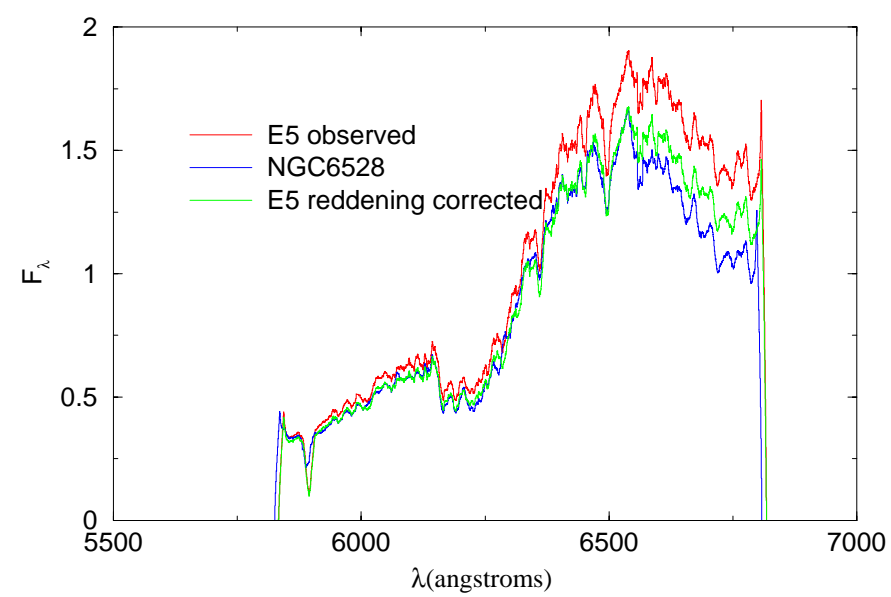

Fig. 1. EROS-BLG-2000-5 (upper spectrum) and NGC 6528: I-2 (lower spectrum), heavily smoothed to show the flux distribution of the two stars. The reddening difference between the two stars is $\Delta E(B-V) \sim 0.2$, as can be seen in the corrected spectrum, relative to NGC 6528: I-2 (middle dotted spectrum). 
In order to derive the flux difference between EROSBLG-2000-5 and NGC 6528: I-2, we estimated slit losses on the estimated flux difference, plus the difference in exposure time. The flux difference is of a factor $\sim 7.4$ at $5870 \AA$ which means that, since NGC 6528: I-2 has $V=15.84$ (Ortolani et al. 1995), the observed star should be about $2.17 \mathrm{mag}$ fainter, or $V \approx 18.0$, therefore magnified by $0.4 \mathrm{mag}$. This agrees with the EROS photometry, which gives: $V=18.4, V-R=1.2$. Note that at peak magnitude on June 14 it had $V=15.6, V-R=1.2$. This shows no blending effect, given that the colour did not change, consistent with the spectrum.

\subsection{Reddening}

The reddening difference between the two stars is also apparent: EROS-BLG-2000-5 seems to be more affected than NGC 6528: I-2. Taking advantage of the long baseline an estimate of the relative reddening between EROSBLG-2000-5 and NGC 6528: I-2 was derived, amounting to $\Delta E(B-V) \sim 0.2$, as also shown in Fig. 1, where the flux corrected spectrum of EROS-BLG-2000-5 relative to NGC 6528: I-2 is the middle dotted spectrum (using Howarth 1983 for the interstellar extinction law).

Dust emission reddening maps of Schlegel et al. (1998) provide in the direction of EROS-BLG-2000-5 $E(B-$ $V)_{\mathrm{FIR}}=1.22$, while in the direction of NGC 6528: I-2, $E(B-V)_{\mathrm{FIR}}=0.77$. These values are upper limits for the stars, since they correspond to the total column density of dust in those directions. In particular, Dutra \& Bica (2000) have discussed background and foreground reddening in the direction of star clusters, and for NGC 6528 they found evidence of some dust occurring in the background. Owing to the lower Galactic latitude of EROS-BLG-2000-5, more background dust is expected than for NGC 6528 . We adopt $E(B-V)=0.52$ for NGC 6528 (Barbuy et al. 1998), and as a consequence $E(B-V)=0.72$ for EROS-BLG-2000-5.

\subsection{Effective temperature}

The EROS project colours are not suitable for effective temperature derivations, since the calibrations in wellknown systems still require further efforts. A temperature value of $T_{\text {eff }}=3800 \mathrm{~K}$ is adopted for EROS-BLG-2000-5, given the presence of $\mathrm{TiO}$ bands, and overall similarity of its spectrum with that of NGC 6528: I-2, for which $T_{\text {eff }} \approx 3600-3800 \mathrm{~K}$, based on HST VI colours (Coelho et al. 2001).

\subsection{Metallicity and abundances}

Equivalent widths of lines measured using IRAF are given in Table 2. Curves of growth of Fe I, Fe II and Ti I are shown in Figs. 2a,b and 3. The gravity of $\log g=$ 1.0 was obtained from a compromise fit of FeI and FeII curves of growth; we obtain $[\mathrm{Fe} / \mathrm{H}]=-0.3$ from FeI and
Table 2. List of FeI and Fe II lines.

\begin{tabular}{|c|c|c|c|c|}
\hline Species & $\lambda(\AA)$ & $\chi_{\mathrm{ex}}(\mathrm{eV})$ & $\log g f$ & $E W(\mathrm{~m} \AA)$ \\
\hline Fe I & 5853.161 & 1.48 & -5.13 & 126. \\
\hline Fe I & 5855.086 & 4.61 & -1.52 & 89. \\
\hline Fe I & 5859.596 & 4.55 & -0.60 & 93. \\
\hline $\mathrm{Fe} I$ & 5861.111 & 4.28 & -2.45 & 22. \\
\hline Fe I & 5862.368 & 4.55 & -0.39 & 84. \\
\hline Fe I & 5927.797 & 4.65 & -1.09 & 61. \\
\hline $\mathrm{Fe} I$ & 5930.191 & 4.65 & -0.23 & 104. \\
\hline Fe I & 5952.726 & 3.98 & -1.44 & 74. \\
\hline Fe I & 5956.706 & 0.86 & -4.605 & 202. \\
\hline $\mathrm{Fe} \mathrm{I}$ & 5969.578 & 4.28 & -2.73 & 34. \\
\hline Fe I & 5987.070 & 4.79 & -0.15 & 93. \\
\hline $\mathrm{Fe} I$ & 6003.022 & 3.88 & -1.12 & 110. \\
\hline $\mathrm{Fe} I$ & 6012.77 & 4.56 & -4.2 & 69. \\
\hline $\mathrm{Fe} I$ & 6054.080 & 4.37 & -2.33 & 39. \\
\hline $\mathrm{Fe} I$ & 6056.013 & 4.73 & -0.46 & 78. \\
\hline $\mathrm{Fe} I$ & 6078.499 & 4.79 & -0.40 & 172. \\
\hline $\mathrm{Fe} \mathrm{I}$ & 6079.02 & 4.65 & -0.99 & 24. \\
\hline Fe I & 6082.72 & 2.22 & -3.57 & 113. \\
\hline $\mathrm{Fe} \mathrm{I}$ & 6151.62 & 2.18 & -3.30 & 157. \\
\hline $\mathrm{Fe} I$ & 6157.733 & 4.07 & -1.26 & 148. \\
\hline Fe I & 6159.38 & 4.61 & -1.85 & 120. \\
\hline $\mathrm{Fe} \mathrm{I}$ & 6165.363 & 4.14 & -1.47 & 106. \\
\hline $\mathrm{Fe} I$ & 6187.995 & 3.94 & -1.72 & 73. \\
\hline Fe I & 6200.398 & 2.61 & -2.437 & 115. \\
\hline Fe I & 6246.327 & 3.60 & -0.88 & 102. \\
\hline $\mathrm{Fe} I$ & 6252.565 & 2.4 & -1.687 & 180. \\
\hline Fe I & 6270.231 & 2.86 & -2.61 & 128. \\
\hline $\mathrm{Fe} I$ & 6297.799 & 2.22 & -2.74 & 170. \\
\hline $\mathrm{Fe} \mathrm{I}$ & 6301.508 & 3.65 & -0.60 & 140. \\
\hline Fe I & 6303.461 & 4.32 & -2.67 & 23. \\
\hline $\mathrm{Fe} I$ & 6315.314 & 4.14 & -1.2 & 119. \\
\hline $\mathrm{Fe} I$ & 6322.694 & 2.59 & -2.426 & 160. \\
\hline $\mathrm{Fe} I$ & 6355.035 & 2.84 & -2.29 & 148. \\
\hline Fe I & 6358.687 & 0.86 & -4.47 & 227. \\
\hline Fe I & 6411.658 & 3.65 & -0.72 & 153. \\
\hline $\mathrm{Fe} I$ & 6419.96 & 4.73 & -0.20 & 116. \\
\hline $\mathrm{Fe} I$ & 6430.856 & 2.18 & -2.01 & 272. \\
\hline Fe I & 6481.878 & 2.28 & -2.98 & 158. \\
\hline $\mathrm{Fe} I$ & 6498.95 & 0.96 & -4.63 & 142. \\
\hline $\mathrm{Fe} I$ & 6518.37 & 2.83 & -2.40 & 127. \\
\hline Fe I & 6574.25 & 0.99 & -5.01 & 171. \\
\hline Fe I & 6597.57 & 4.77 & -0.97 & 67. \\
\hline $\mathrm{Fe} I$ & 6627.56 & 4.55 & -1.68 & 59. \\
\hline Fe I & 6677.997 & 2.69 & -1.42 & 228. \\
\hline $\mathrm{Fe} I$ & 6699.14 & 4.59 & -2.12 & 60. \\
\hline $\mathrm{Fe} I$ & 6713.044 & 4.79 & -1.48 & 58. \\
\hline $\mathrm{Fe} \mathrm{I}$ & 6725.36 & 4.10 & -2.23 & 29. \\
\hline $\mathrm{Fe} I \mathrm{II}$ & 5425.26 & 3.20 & -3.37 & 39. \\
\hline Fe II & 5534.85 & 3.24 & -2.93 & 51. \\
\hline $\mathrm{Fe} I I$ & 5991.38 & 3.15 & -3.56 & 60. \\
\hline
\end{tabular}


Table 3. List of V I, Ti I, Y II, Ba II, Eu II, Al I lines.

\begin{tabular}{lllll}
\hline Species & $\lambda(\AA)$ & $\chi_{\text {ex }}(\mathrm{eV})$ & $\log g f$ & $E W(\mathrm{~m} \AA)$ \\
\hline V I & 6039.74 & 1.06 & -0.65 & 164. \\
V I & 6081.45 & 1.05 & -0.58 & 168. \\
V I & 6090.22 & 1.08 & -0.06 & 188. \\
V I & 6111.65 & 1.04 & -0.72 & 19. \\
V I & 6119.53 & 1.06 & -0.32 & 150. \\
V I & 6128.33 & 1.05 & -2.30 & 96. \\
V I & 6135.07 & 1.35 & -1.79 & 87. \\
V I & 6150.15 & 0.30 & -1.78 & 235. \\
V I & 6199.19 & 0.29 & -1.30 & 171. \\
V I & 6224.51 & 0.29 & -2.01 & 87. \\
V I & 6233.20 & 0.28 & -2.07 & 214. \\
V I & 6274.66 & 0.27 & -1.67 & 148. \\
V I & 6285.17 & 0.28 & -1.51 & 155. \\
Ti I & 5866.46 & 1.07 & -0.84 & 358. \\
Ti I & 5918.55 & 1.07 & -1.46 & 153. \\
Ti I & 5941.76 & 1.05 & -1.51 & 182. \\
Ti I & 5965.84 & 1.88 & -0.41 & 198. \\
Ti I & 5978.55 & 1.87 & -0.50 & 112. \\
Ti I & 6064.63 & 1.05 & -1.94 & 158. \\
Ti I & 6091.18 & 2.27 & -0.42 & 127. \\
Ti I & 6092.82 & 1.89 & -1.38 & 69. \\
Ti I & 6126.22 & 1.07 & -1.43 & 170. \\
Ti I & 6303.77 & 1.44 & -1.57 & 121. \\
Y II & 6613.73 & 1.75 & -1.27 & 143. \\
Ba II & 5853.69 & 0.60 & -1.01 & 154. \\
Ba II & 6141.73 & 0.70 & -0.08 & 218. \\
Ba II & 6496.91 & 0.60 & -0.38 & 211. \\
Eu II & 6645.13 & 1.38 & 0.20 & 35. \\
Al I & 6696.03 & 3.14 & -1.34 & 152. \\
Al I & 6698.67 & 3.14 & -1.64 & 122. \\
\hline & & & & \\
\hline
\end{tabular}

$[\mathrm{Fe} / \mathrm{H}] \sim 0.0$ from FeII, where the latter is more uncertain due to the small number of lines. Final parameters adopted are $T_{\text {eff }}=3800 \mathrm{~K}, \log g=1.0,[\mathrm{Fe} / \mathrm{H}]=-0.3$, $v_{t}=1.5 \mathrm{~km} \mathrm{~s}^{-1}$. We estimate errors of \pm 200 in $T_{\text {eff }}, \pm 0.5$ in $\log g$ and \pm 0.3 in $[\mathrm{Fe} / \mathrm{H}]$.

A check on the FeII lines was carried out by means of synthetic spectra calculations, since the lines are weak and possible blends of $\mathrm{TiO}$ tend to artificially increase the measured equivalent widths. In Figs. 4 and 5 the spectral region containing the [OI] $6300.311 \AA$ line, blended with strong TiO bands, is shown for EROS-BLG-2000-5. The telluric lines were eliminated by dividing the sample spectra by that of a hot star with high rotation velocity, and observed with the same slit width. In Fig. 4 we also show that $\mathrm{TiO}$ bands considerably lower the continuum, where $\mathrm{TiO}$ bands are computed with $[\mathrm{O} / \mathrm{Fe}]=[\mathrm{Ti} / \mathrm{Fe}]=$ $-0.2,0.0$ and +0.2 .
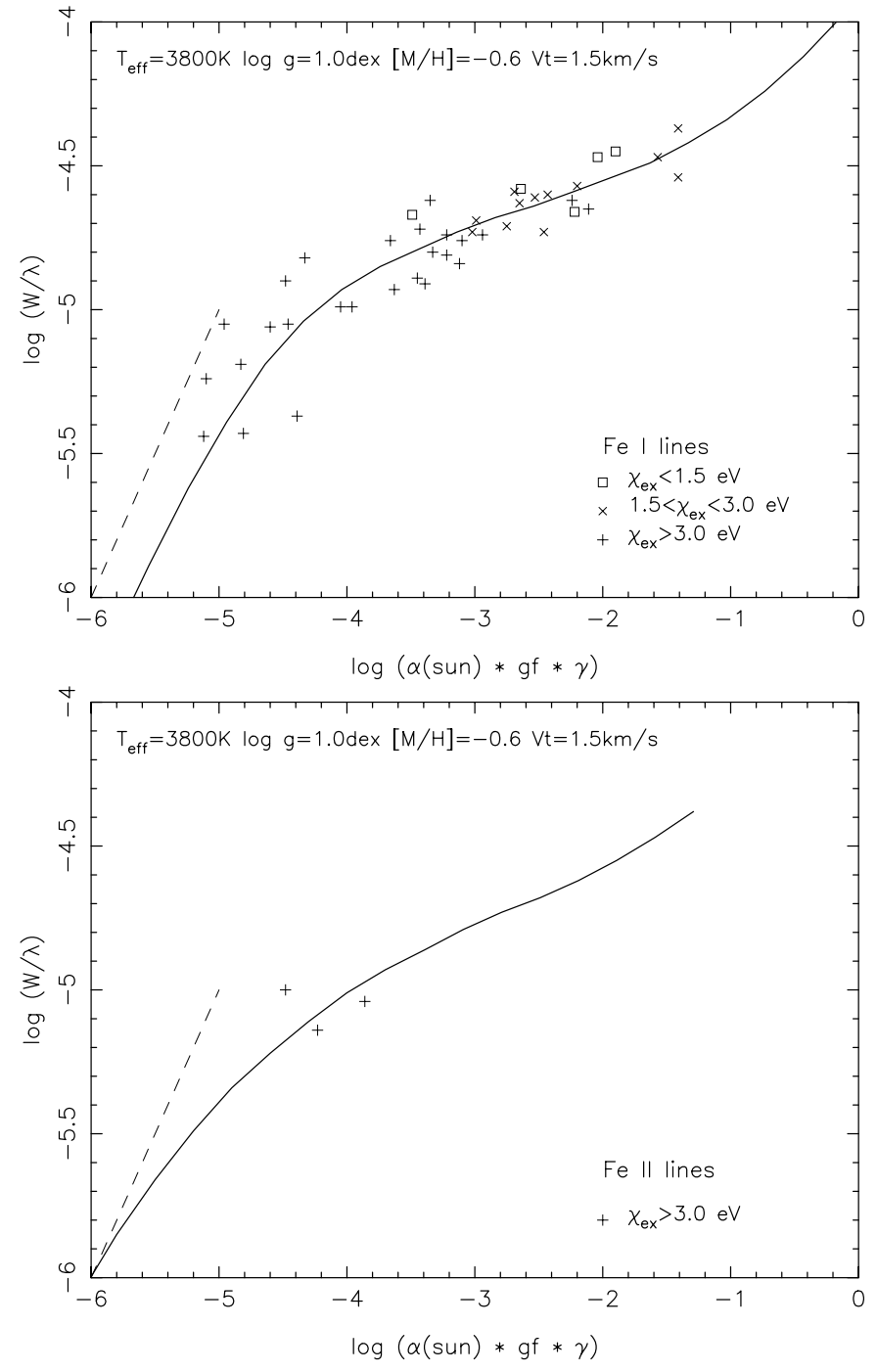

Fig. 2. Curves of growth of Fe I and Fe II. Symbols: $\chi_{\mathrm{ex}}<$ $1.5 \mathrm{eV}$ (open squares), $1.5<\chi_{\mathrm{ex}}<3.0 \mathrm{eV}(\mathrm{x}), \chi_{\mathrm{ex}}>3.0 \mathrm{eV}(+)$.

The oxygen abundances are estimated by taking into account the molecular dissociation equilibrium, since in this cool temperature range $\mathrm{CO}$ locks a large fraction of oxygen atoms. Considering the continuum uncertainties, we conclude that the oxygen abundance can be estimated as $[\mathrm{O} / \mathrm{Fe}] \approx 0.0 \pm 0.2$.

\subsection{Distance}

For NGC 6528 a distance to the Sun of $d_{\odot} \approx 7.8 \mathrm{kpc}$ (Barbuy et al. 1998) was found. Using the reddening value of $E(B-V)=0.52$ for the cluster, and the observed $V$ magnitude of the giant NGC 6528: I-2 of $V=$ 15.84, we derive an absolute magnitude $M_{\mathrm{V}}=-0.3$ for this star.

For EROS-BLG-2000-5 the non-lensed magnitude is $V=18.4$, and we assume it has the same intrinsic luminosity as NGC 6528: I-2; taking into account $E(B-V)=0.72$ (Sect. 3.1), together with a total-to-selective absorption 


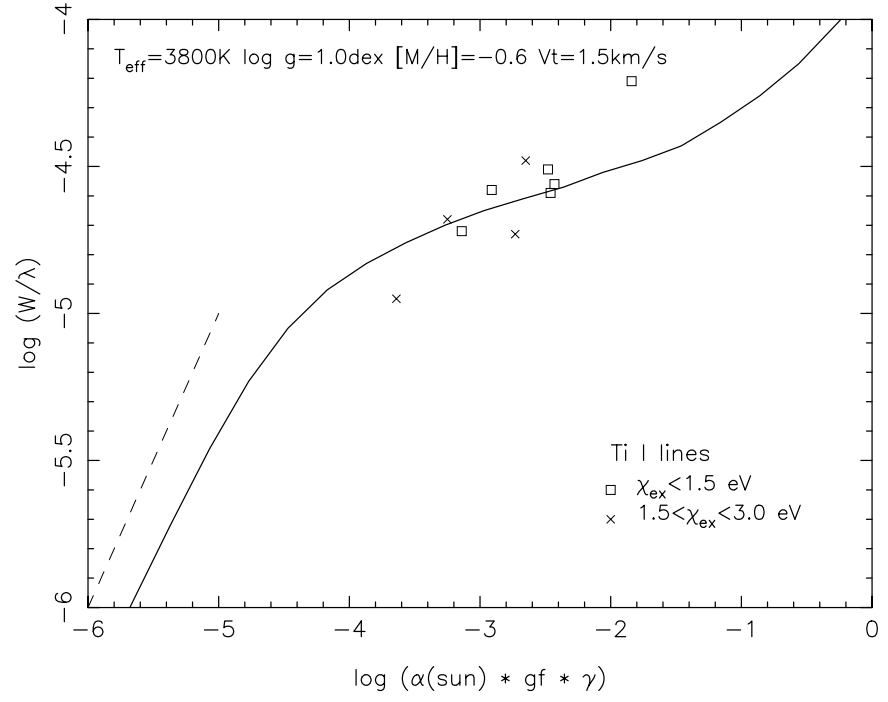

Fig. 3. Curve of growth of Ti I. Same symbols as in Fig. 2.

$R=3.2$, we obtain a distance modulus of $(m-M)_{\circ}=$ 16.4. This would place the star near the solar circle on the far side of the Galaxy. In this case it would be at $600 \mathrm{pc}$ from the plane, compatible with a location of an old disk giant. Nevertheless, it may be more evolved than the star in NGC 6528: I-2 (even with a comparable temperature, it can be more luminous); assuming that such a possible difference would be of $1 \mathrm{mag}$, this would place the star at about $10 \mathrm{kpc}$ from the Sun and a height of $400 \mathrm{pc}$ from the plane. In this case it would be a bulge star on the far side. It is clear that the uncertainty in the distance is very large. An important constraint is given by the high radial velocity (Sect. 3), which is definitely consistent and supports a bulge membership.

A final calibration of the EROS colours or direct photometry can clarify this issue.

\section{Conclusions}

We have carried out a high resolution spectrophotometric analysis of the bulge K giant star EROS-BLG-2000-5 while it was magnified due to microlensing. The derived stellar parameters are: $T_{\text {eff }}=3800 \pm 200 \mathrm{~K}, \log g=1.0 \pm 0.5$, $[\mathrm{Fe} / \mathrm{H}]=-0.3 \pm 0.3,[\mathrm{O} / \mathrm{Fe}] \approx 0.0 \pm 0.2$.

It is interesting to note that the metallicity is close to the mean value found by McWilliam \& Rich (1994) for bulge giants in Baade's Window. Within uncertainties of distances it would be compatible either with a far side old disk giant, or a far side bulge giant.

It would be important to make efforts to calibrate the EROS magnitudes to final values in standard systems and to pursue this kind of microlensing surveys, since it provides a powerful means of observing faint distant objects, in particular in the bulge direction where these events are much more frequent.

Acknowledgements. DM is supported by Fondecyt 1990440. $\mathrm{BB}$ and $\mathrm{EB}$ acknowledge partial financial support from

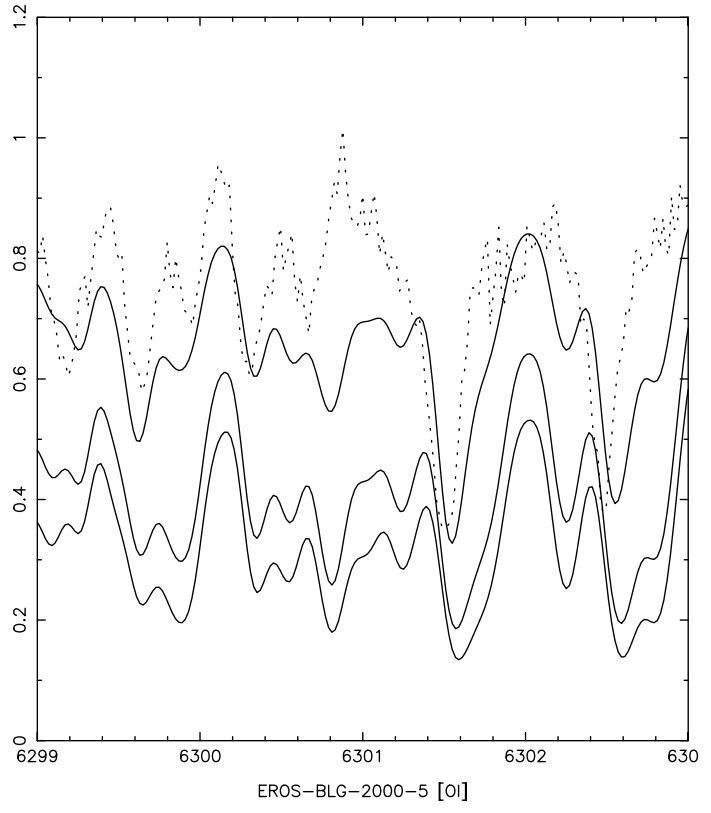

Fig. 4. [OI] line region spectrum of the lensed star EROS-BLG2000-5 (dotted line) compared to synthetic spectra computed with $[\mathrm{O} / \mathrm{Fe}]=[\mathrm{Ti} / \mathrm{Fe}]=-0.2,0.0$ and +0.2 , showing the effect of the $\mathrm{TiO}$ bands on the continuum level.

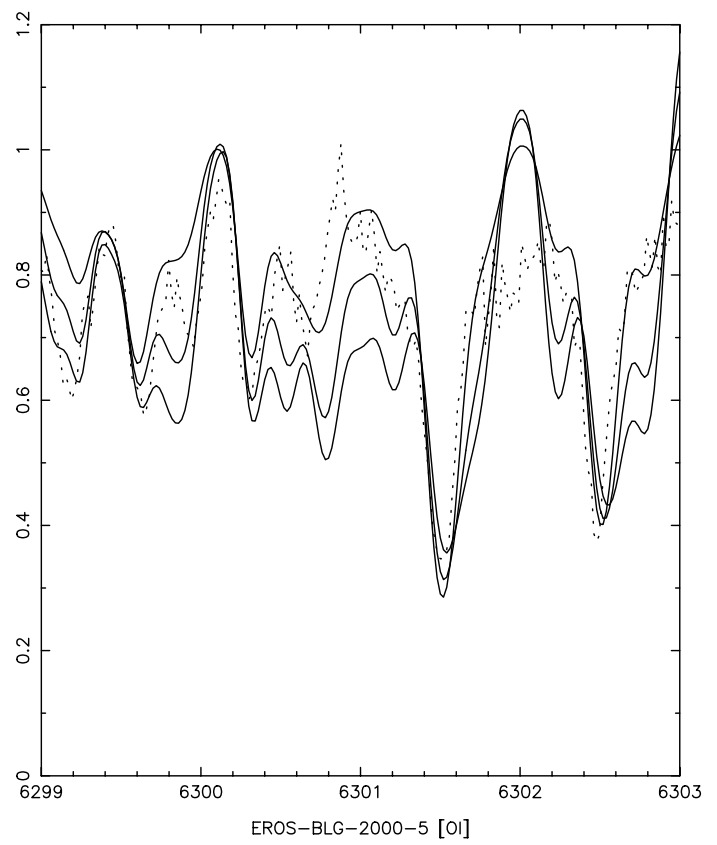

Fig. 5. [OI] line region spectrum of EROS-BLG-2000-5 (dotted line) compared to synthetic spectra computed with $[\mathrm{O} / \mathrm{Fe}]=$ $[\mathrm{Ti} / \mathrm{Fe}]=-0.2,0.0$ and +0.2 , and normalized to the observed spectrum, aiming to fit the [OI]6300.311 $\AA$ line.

the Brazilian agencies $\mathrm{CNPq}$ and Fapesp. SO acknowledges the Italian Ministero dell'Università e della Ricerca Scientifica e Tecnologica (MURST) under the program on "Stellar Evolution" (Italy). KHC performed this work under the auspices of the U.S. Department of Energy by the University of California, Lawrence Livermore National Laboratory under contract No. W-7405-Eng-48. 


\section{References}

Albrow, M., An, J., Beaulieu, J.-P., et al. (PLANET collaboration) 2000, ApJ, 550, L173

An, J., Albrow, M., Beaulieu, P., et al. 2001, ApJ, submitted [astro-ph/0110095]

Balester, P., Modigliani, A., Boitquin, O., et al. 2000, The Messenger, 101, 31

Barbuy, B., Bica, E., \& Ortolani, S. 1998, A\&A, 333, 117

Benetti, S., Pasquini, L., \& West, R. 1995, A\&A, 294, L37

Castro, S., Pogge, R. W., Rich, R. M., et al. 2001, ApJ, 548, L197 [astro-ph/0101025]

Coelho, P., Barbuy, B., Perrin, M.-N., et al. 2001, A\&A, 376, 136

Dutra, C., \& Bica, E. 2000, A\&A, 359, 347

Harris, W. E. 1996, AJ, 112, 1487 (H96)
Howarth, I. D. 1983, MNRAS, 203, 301

Ibata, R. A., \& Gilmore, G. 1995, MNRAS, 275, 605

Ibata, R .A., Gilmore, G., \& Irwin, M. J. 1995, AJ, 277, 781

Lennon, D. J., Mao, S., Fuhrmann, K., Gehren, T. 1996, ApJ, 471, L23

McWilliam, A., \& Rich, R. M. 1994, ApJS, 91, 749

Minniti, D. 1996, ApJ, 459, 579

Minniti, D., Vandehei, T., Cook, K. H., Griest, K., \& Alcock, C. 1998, ApJ, 499, L175

Ortolani, S., Renzini, A., Gilmozzi, R., et al. 1995, Nature, 377, 701

Reid, M. 1993, ARA\&A, 31, 345

Rieke, G. H., \& Lebofsky, M. J. 1985, ApJ, 288, 618

Schlegel, D. J., Finkbeiner, D. P., \& Davis, M. 1998, ApJ, 500, 525

van den Bergh, S., \& Younger, F. 1979, AJ, 84, 1305 